\title{
Cloud-Enabled Differentially Private Multi-Agent Optimization with Constraints
}

\author{
Matthew T. Hale and Magnus Egerstedt
}

\begin{abstract}
We present an optimization framework for solving multi-agent nonlinear programs subject to inequality constraints while keeping the agents' state trajectories private. Each agent has an objective function depending only upon its own state and the agents are collectively subject to global constraints. The agents do not directly communicate with each other but instead route messages through a trusted cloud computer. The cloud computer adds noise to data being sent to the agents in accordance with the framework of differential privacy in order to keep each agent's state trajectory private from all other agents and any eavesdroppers. This private problem can be viewed as a stochastic variational inequality and is solved using a projection-based method for solving variational inequalities that resembles a noisy primal-dual gradient algorithm. Convergence of the optimization algorithm in the presence of noise is proven and a quantifiable trade-off between privacy and convergence is extracted from this proof. Simulation results are provided that demonstrate numerical convergence for both $\epsilon$-differential privacy and $(\epsilon, \delta)$-differential privacy.
\end{abstract}

\section{INTRODUCTION}

Optimization problems spread across teams of agents arise naturally in several fields, including communications [1], [2], robotics [3], [4], machine learning [5], sensor networks [6], [7], and smart power grids [8], [9], [10]. Correspondingly, a variety of approaches have been developed that solve problems with a wide variety of formulations. For example, [11] allows for distributed

The authors are with the School of Electrical and Computer Engineering, Georgia Institute of Technology, Atlanta, GA 30332, USA. Email: \{matthale, magnus\}@gatech. edu. Research supported in part by the NSF under Grant CNS-1239225. 
optimization of non-differentiable objectives with time-varying communication links, [12] considers a similar problem formulation in which communication links fail over time, and [13] uses a distributed Newton method to solve dynamic network utility maximization problems. Many other problem types and solution schemes exist in the literature, and a broad exposition of results can be found in [14].

In some cases multi-agent optimization is done using sensitive user data. A concrete example of such a case comes from smart power grids. In smart power grids, homeowners share their power usage information with others on the grid to allow network management (e.g., frequency regulation [10]) and to minimize their own power costs. In some cases, the granular power usage data shared in smart grids can be used to infer sensitive details of users' personal lives [15], [16]. In particular, smart grid data can "provide a detailed breakdown of energy usage over a long period of time, which can show patterns of use," [16, Page 15, Item 16]. Further, given these patterns, "[p]rofiles can thus be developed and then applied back to individual households and individual members of these households," [16. Page 15, Item 18]. These usage patterns in turn "could reveal personal details about the lives of consumers, such as their daily schedules," [15, Page 2, Paragraph 5].

It is precisely the deduction of such patterns that we wish to prevent in the context of multiagent optimization. Based on the potentially revealing nature of some user data, we seek to optimize while protecting sensitive user data both from eavesdroppers and other agents in the network. In some sense, privacy and optimization are competing objectives in that agents who only seek to optimize may freely share their states with others in the network, while agents concerned only with privacy may be inclined to share no information at all. To privately optimize, then, we must strike a balance between these two different, competing objectives.

One approach to privacy that has recently seen widespread use is differential privacy. Differential privacy was originally established in the database literature and keeps sensitive database entries private when a database is queried by adding noise to the result of that query [17], [18]. The authors of [19], [20] survey some of the important developments in this vein. Differential privacy has been adapted to dynamical systems in order to keep sensitive inputs private from 
an adversary observing a system's outputs [21]. A dynamical system is differentially private if inputs that are close in the input space produce outputs that have similar probability distributions; these notions will be made precise in Section III

It is the dynamical systems notion of differential privacy that we apply to keep agents' state trajectories private while optimizing. One appealing aspect of differential privacy is its resilience to post-processing, which allows for arbitrary processing of private information without the threat of its privacy guarantees being weakened [21, Theorem 1]. Differential privacy is also robust to arbitrary side information, meaning that an adversary cannot weaken differential privacy by much through using information gleaned from another source [22].

There has already been some work on enforcing differential privacy in optimization. In [23] linear programs are solved in a framework that allows for keeping objective functions or constraints private. The authors of [24] consider a similar setting wherein linearly constrained problems with affine objectives are solved while keeping the objective functions private. In the multi-agent setting, [25] solves distributed consensus-type problems while keeping the agents' objective functions private, while [26] solves similar problems while keeping each agent's initial state private.

In this paper we solve non-linear programs wherein each agent's state trajectory is sensitive information and the agents therefore seek to protect their exact state trajectories from other agents and any eavesdroppers. To protect these sensitive data, a trusted cloud computer is used that performs certain computations upon information it receives from the agents, makes the results of those computations private by adding noise to them, and then sends the private results to each agent. Each agent then updates its state locally using the information it received from the cloud, and this process of sharing and updating information is repeated.

Our motivation for developing a mixed centralized/decentralized algorithm is inspired by the prominence of cloud computing in many real-world applications. A survey of existing cloud applications is given in [27], and that reference elaborates on the scalability of the cloud and its ability to coordinate many mobile devices. It is precisely these features of the cloud that make it an attractive choice here. In this paper, the cloud, viewed as a central aggregator, is an integral 
part of the optimization process, and we leverage its scalability to aggregate ensemble-level information, perform computations upon that data in a private manner, and then distribute these private results to the agents.

The privacy implementation in this paper differs from the aforementioned references on private optimization in several key ways. We are interested in solving problems in which the agents collectively run an on-line optimization algorithm collaboratively by sharing (private functions of) sensitive information. In the problems we consider, each iteration of the optimization algorithm determines each agent's next state. That is, the iterates of the optimization algorithm are the agents' states, and it is each agent's desire to keep its state trajectory private to protect information about its behavior. Accordingly, while the above references on private optimization keep other problem data private, here we must keep entire trajectories of states private while optimizing. In addition, we incorporate both nonlinear inequality constraints and set constraints, which, to our knowledge, has not been explored in other privacy implementations.

Given the need to optimize while remaining private, encryption alone cannot provide the privacy guarantees that are needed in the problems we examine. In the "upstream" direction, encryption could be used to protect communications sent from the agents to the cloud, provided the cloud could decrypt them. However in the "downstream" direction, when the cloud sends transmissions to the agents, any encrypted messages from the cloud would naturally need to be decrypted by the agents to allow each agent to update its state. While this strategy can protect transmissions of sensitive data from eavesdroppers, having the agents decrypt transmissions from the cloud would expose all agents' sensitive data to each agent in the network, violating the privacy guarantees that are required by each agent. Instead, what is required here is a privacy implementation that protects user data from eavesdroppers and all others in the network, while still making that data useful for optimizing. It is for this reason that we use differential privacy.

A preliminary version of this work appeared in [28]. The current paper adds a proof of convergence, a convergence estimate, quantifies the privacy-convergence trade-off, and provides new numerical results for two different privacy mechanisms. The rest of the paper is organized as follows. Section II lays out the problem to be solved and its method of solution. Next, 
Section III covers the necessary elements of differential privacy and relates them to the setting of optimization. Then Section IV provides a proof of convergence for the optimization algorithm used here and a bound on its convergence, in addition to exploring the trade-off between privacy and convergence. Next, Section V provides simulation results to support the theoretical developments made. Finally, Section VI concludes the paper.

\section{OptimizATION PROBLEM Formulation}

In this section we lay out the problem to be solved. First in Section $[\mathrm{II}-\mathrm{A}$ we lay out the multiagent problem and then, to aid in the exposition of its solution method, formulate an equivalent ensemble problem. Then the solution to that problem will be discussed and, in Section II-B, will be adapted to the cloud-based architecture used here.

\section{A. Problem Overview}

Consider $N$ agents indexed over the set $I:=\{1, \ldots, N\}$, with agent $i$ having state $x_{i} \in \mathbb{R}^{n_{i}}$ for some $n_{i} \in \mathbb{N}$. Agent $i$ seeks to minimize the objective function

$$
f_{i}: \mathbb{R}^{n_{i}} \rightarrow \mathbb{R}
$$

where $f_{i}$ depends only upon $x_{i}$, that is, each agent's objective function has no dependence upon the other agents' states. Using the notation $\nabla_{i} f_{i}:=\frac{\partial f_{i}}{\partial x_{i}}$, we state the following assumption for objective functions.

Assumption 1: The function $f_{i}$ is $C^{1}$ and convex, and $\nabla_{i} f_{i}$ is Lipschitz with constant $L_{i}$ for all $i \in I$.

Assumption 1 allows for a broad class of functions to be used as objective functions, including any $C^{2}$ convex function on a compact, convex domain (cf. Assumption 2 below). Each agent's state is constrained to lie in a given set which we express as

$$
x_{i} \in X_{i} \subset \mathbb{R}^{n_{i}} .
$$

Regarding each set $X_{i}$, we state the following assumption. 
Assumption 2: Each set $X_{i}$ is non-empty, compact, and convex.

In particular Assumption 2 admits box constraints which are common in some multi-agent problems.

Now define the ensemble state vector

$$
x=\left(\begin{array}{c}
x_{1} \\
x_{2} \\
\vdots \\
x_{N}
\end{array}\right) \in \mathbb{R}^{n}
$$

where $n=\sum_{i=1}^{N} n_{i}$. We impose global inequality constraints on the agents by requiring

$$
g(x):=\left(\begin{array}{c}
g_{1}(x) \\
g_{2}(x) \\
\vdots \\
g_{m}(x)
\end{array}\right) \leq 0,
$$

where the above inequality is enforced component-wise, i.e., $g_{j}(x) \leq 0$ for all $j \in J:=$ $\{1, \ldots, m\}$. We now state our assumptions on $g$.

Assumption 3: The function $g: \mathbb{R}^{n} \rightarrow \mathbb{R}^{m}$ is $C^{1}$ and convex. In addition, for $\mathbb{R}^{m}$ and $\mathbb{R}^{n}$ both equipped with the same $p$-norm, the function $g_{x_{j}}:=\frac{\partial g}{\partial x_{j}}$ is Lipschitz continuous with constant $K_{p}^{j}$ for all $j \in J$ with respect to the metric induced by the $p$-norm. In addition the function $g$ is Lipschitz with constant $K_{p}^{g}$ with respect to the same metric.

In this paper we focus on the cases of $p=1$ and $p=2$. Like Assumption 1, Assumption 3 allows for any convex, $C^{2}$ functions to be used for constraints whenever Assumption 2 holds. We also have the following assumption on $g$.

Assumption 4: The constraints satisfy Slater's condition, namely there exists a point $\bar{x} \in X$ such that $g(\bar{x})<0$.

Assumption 4 is commonly enforced in nonlinear programming problems to guarantee that strong duality holds. Under Assumptions 1, 4, we state an ensemble-level optimization problem. 
To do so, we define the ensemble objective

$$
f(x)=\sum_{i=1}^{n} f_{i}\left(x_{i}\right)
$$

and the set

$$
X=\prod_{i=1}^{n} X_{i}
$$

where the product is meant in the Cartesian sense. To fix ideas, we state the following optimization problem that does not yet incorporate privacy; privacy will be formally included in Problem 1 in Section III,

Problem 0.1: (Preliminary; no privacy requirement)

$$
\begin{aligned}
\operatorname{minimize} & f(x) \\
\text { subject to } & g(x) \leq 0 \\
& x \in X .
\end{aligned}
$$

We note here that Problem 0.1 will be solved without having agent $i$ share $f_{i}$ or $X_{i}$ with the other agents or with the cloud because these data are considered sensitive information. Similarly, $g$ is considered sensitive and the cloud does not share $g$ with any of the agents. The Lagrangian associated with Problem 0.1 is

$$
L(x, \mu)=f(x)+\mu^{T} g(x),
$$

where $\mu$ is a vector of Kuhn-Tucker multipliers in the non-negative orthant of $\mathbb{R}^{m}$, denoted $\mathbb{R}_{+}^{m}$. Under Assumptions 1, 2, and 3 a primal solution $\hat{x}$ exists and the set of all primal solutions is non-empty and compact. With the addition of Assumption 4 , a dual solution $\hat{\mu}$ exists and the optimal primal and dual values are equal [29, Proposition 6.4.3]. 
Under Assumptions 1-4, a point $\hat{x}$ solves Problem 0.1 if and only if there exists a point $\hat{\mu} \in \mathbb{R}_{+}^{m}$ such that $(\hat{x}, \hat{\mu})$ is a saddle point of $L$, that is, if and only if the point $(\hat{x}, \hat{\mu})$ satisfies

$$
L(\hat{x}, \mu) \leq L(\hat{x}, \hat{\mu}) \leq L(x, \hat{\mu})
$$

for all $(x, \mu) \in X \times \mathbb{R}_{+}^{m}[29$, Proposition 6.2.4]. It is as saddle points of $L$ that we seek solutions $(\hat{x}, \hat{\mu})$ to Problem 0.1 .

Toward that end, we next define the symbols

$$
L_{x}:=\frac{\partial L}{\partial x}, L_{\mu}:=\frac{\partial L}{\partial \mu}, g_{x}:=\frac{\partial g}{\partial x}, \text { and } f_{x}:=\frac{\partial f}{\partial x}
$$

and define the map

$$
G(x, \mu)=\left(\begin{array}{r}
L_{x}(x, \mu) \\
-L_{\mu}(x, \mu)
\end{array}\right)
$$

In what follows, it is necessary for $G$ to be a Lipschitz mapping. Though the maps $f_{x}$ and $g_{x}$ are Lipschitz by Assumptions 1 and 3, $G$ itself cannot be shown to be Lipschitz because its domain, $X \times \mathbb{R}_{+}^{m}$, is unbounded by virtue of $\mathbb{R}_{+}^{m}$ being unbounded. To rectify this situation, we use Equation (1) to find a non-empty, convex, compact set containing $\hat{\mu}$ as was done in [30]. From the second inequality in Equation (1) we have

$$
f(\hat{x})+\hat{\mu}^{T} g(\hat{x}) \leq f(\bar{x})+\hat{\mu}^{T} g(\bar{x})
$$

for $\bar{x}$ the Slater point as defined in Assumption 4. By the complementary slackness condition we have

$$
f(\hat{x}) \leq f(\bar{x})+\hat{\mu}^{T} g(\bar{x})
$$

Rearranging we find

$$
\sum_{j=1}^{m} \hat{\mu}_{j} \leq \frac{f(\bar{x})-f(\hat{x})}{\min _{1 \leq j \leq m}\left\{-g_{j}(\bar{x})\right\}} \leq \frac{f(\bar{x})-f\left(x^{*}\right)}{\min _{1 \leq j \leq m}\left\{-g_{j}(\bar{x})\right\}}
$$


where $x^{*} \in \underset{x \in X}{\arg \min } f(x)$. We then define the set

$$
\mathbb{M}:=\left\{\mu \in \mathbb{R}_{+}^{m}:\|\mu\|_{1} \leq \frac{f(\bar{x})-f\left(x^{*}\right)}{\min _{1 \leq j \leq m}\left\{-g_{j}(\bar{x})\right\}}\right\}
$$

which is non-empty, compact, and convex by definition, and which contains $\hat{\mu}$. For economy of notation, we define the symbols $Z:=X \times \mathbb{M}$ and $\hat{z}:=(\hat{x}, \hat{\mu})$, and we will use $z:=(x, \mu)$ to denote an arbitrary point in $Z$.

Since $L(\cdot, \mu)$ is convex for all $\mu \in \mathbb{M}$ and $L(x, \cdot)$ is concave for all $x \in X$, we see that $G$ is monotone [31, Theorem A]. Under Assumptions 1-4, a primal-dual pair $(\hat{x}, \hat{\mu})$ is a saddle point of $L$ if and only if it solves the following variational inequality (VI) [32, Corollary 11.1].

Problem 0.2: (VI formulation; no privacy requirement) Find a point $\hat{z} \in Z$ such that

$$
\langle z-\hat{z}, G(\hat{z})\rangle \geq 0
$$

for all $z \in Z$.

Further discussion on the equivalence of Problems 0.1] and 0.2 is given in [33], Sections 1.3.1, 1.3.2, and 1.4.1. Privacy is formally added to Problem 0.2 in the statement of Problem 1 in Section III.

We will use the notation $V I(K, F)$ to denote the generic problem of finding a point $x \in K$ such that

$$
\langle y-x, F(x)\rangle \geq 0
$$

for all $y \in K$, and we will use the notation $S O L(K, F)$ to denote the solution set of $V I(K, F)$. The symbols $Z$ and $G$ refer to the specific problem under consideration in this paper so that Problem 0.2 is denoted $\operatorname{VI}(Z, G)$ and its solution set is $\operatorname{SOL}(Z, G)$. It is in the setting of variational inequalities that we will proceed and we focus on solving Problem 0.2 with the understanding that its solutions also solve Problem 0.1 .

For a compact set $K$ and a monotone map $F$, one method of solving the variational inequality $V I(K, F)$ is using a projection method with an iterative Tikhonov regularization as was done 
for deterministic variational inequalities in [34] and for stochastic variational inequalities in [35]; these methods regularize the earlier Goldstein-Levitin-Polyak method for solving such problems [36], [37]. The basic principle underlying these methods is that a point in $S O L(K, F)$ can be approached iteratively with $F$ specifying the direction in which to move at each iteration. To endow this procedure with greater numerical stability and, as will be shown, robustness to noise, the $k^{\text {th }}$ iteration specified in [34], [35] instead uses the direction specified by $F+\alpha_{k} I$ with $I$ the identity map, $\alpha_{k}>0$, and $\alpha_{k} \rightarrow 0$. When $F$ is monotone, each map $F+\alpha_{k} I$ is strongly monotone so that $\operatorname{SOL}\left(K, F+\alpha_{k} I\right)$ is a singleton. Letting $\xi_{k}$ denote the (unique) element of $\operatorname{SOL}\left(K, F+\alpha_{k} I\right)$, for $\alpha_{k}>0$ and $\alpha_{k} \rightarrow 0$ we have $\xi_{k} \rightarrow y_{0}$ where $y_{0}$ is the least-norm element of $\operatorname{SOL}(K, F)$ (which itself is non-empty because $K$ is compact and $F$ is monotone).

Given an initial point $z(0) \in Z$, the deterministic form of the regularized method to solve $V I(Z, G)$ is given below in Algorithm 1 .

Algorithm 1: Given a point $z(0) \in Z$, apply the update law

$$
\begin{aligned}
& z(k+1)=\Pi_{Z}\left[z(k)-\gamma_{k}\left(G(z(k))+\alpha_{k} z(k)\right)\right] \\
& =\Pi_{Z}\left[\left(\begin{array}{c}
x(k)-\gamma_{k}\left(f_{x}(x(k))+g_{x}(x(k))^{T} \mu(k)+\alpha_{k} x(k)\right) \\
\mu(k)+\gamma_{k}\left(g(x(k))-\alpha_{k} \mu(k)\right)
\end{array}\right)\right]
\end{aligned}
$$

until a fixed point $\hat{z}$ is reached.

Here $\alpha_{k}$ is the regularization parameter at timestep $k$ and $\gamma_{k}$ is the step-size at the same timestep. In Section III we will use Algorithm 1 to solve a private optimization problem, and in Section IV we provide hypotheses on $\gamma_{k}$ and $\alpha_{k}$ sufficient for convergence. Currently we show the applicability of this style of solution to the cloud architecture mentioned above.

\section{B. Communications}

If we separate the update law in Algorithm 1 to examine the per-agent (primal) update law, we find that agent $i$ executes

$$
x_{i}(k+1)=\Pi_{X_{i}}\left[x_{i}(k)-\gamma_{k}\left(\nabla_{i} f_{i}\left(x_{i}(k)\right)+g_{x_{i}}(x(k))^{T} \mu(k)+\alpha_{k} x_{i}(k)\right)\right] \text {. }
$$


The only terms on the right-hand side of this update law that contain information from other agents are $g_{x_{i}}(x(k))$ and $\mu(k)$. Though $g_{x_{i}}$ is a function of all states in the network, the agents do not send their states to each other directly to allow for its computation because doing so may reveal sensitive information. Instead, every agent sends its state to a trusted cloud computer which computes $g_{x_{i}}(x(k))$ for every $i \in I$. Because no agent has every agent's state value, no agent can compute $\mu(k)$ (cf. Equation (2)) and therefore the cloud computes $\mu(k)$ as well using the update law

$$
\mu(k+1)=\Pi_{\mathbb{M}}\left[\mu(k)+\gamma_{k}\left(g(x(k))-\alpha_{k} \mu(k)\right)\right] .
$$

Then, to use Algorithm 1 with this architecture, the cloud sends (private forms of) $g_{x_{i}}(x(k)$ ) and $\mu(k)$ to agent $i$; the modifications to these quantities to make them private are covered in Section III. The cloud is assumed to be a powerful computer capable of carrying out these calculations quickly so that they reliably arrive at the agents in a timely fashion.

With this communications scheme, at timestep $k$ four actions occur. First, agent $i$ sends $x_{i}(k)$ to the cloud and the cloud assembles all agents' states into the vector $x(k)$. Second, the cloud computes $\mu(k)$ and $g_{x_{i}}(x(k))$ for all $i \in I$ in a differentially private way. Third, the cloud sends a private form of $g_{x_{i}}(x(k))^{T} \mu(k)$ to agent $i$. Fourth, agent $i$ computes $x_{i}(k+1)$ while the cloud simultaneously computes $\mu(k+1)$, and then this sequence of communications and computations is repeated. Because this happens at every timestep, information in the network is always synchronized when computations occur and there is no disagreement between the agents or cloud as to what the value of a particular state is. As a result, the computations that are spread across the network in this manner produce identical results to Algorithm 1, and the ensemble problem is, mathematically, equivalent to the cloud-based multi-agent problem.

For simplicity, the forthcoming analysis will be carried out in the ensemble setting. Despite the mathematical equivalence between the multi-agent and ensemble approaches, the advantage of the cloud-based approach in practice is that it allows for each agent's state trajectory to be kept private while the ensemble approach does not. 


\section{PRIVATE OptimizATION}

Differential privacy originates in the database literature in computer science and was originally designed to keep individual entries of a database private [20]. It has recently been extended to the setting of dynamical systems in [21]. Differential privacy offers a formal definition of privacy as well as resilience to post-processing and robustness to side information. This resilience to post-processing prevents an adversary from weakening the guarantees of differential privacy by performing post-hoc calculations on private information. Robustness to side information guarantees that an adversary cannot use information it has gleaned from an alternate source to fully defeat differential privacy. Below we first review differential privacy, then give a formal private optimization problem statement, and finally discuss applying privacy to Problem 0.2 .

\section{A. Differentially Private Systems}

Let there be $N$ input signals to a system, each contributed by some user. The $i^{\text {th }}$ input signal is denoted $u_{i}$ and is contained in the set $\tilde{\ell}_{p_{i}}^{s_{i}}$, namely the space of sequences of $s_{i}$-vectors equipped with the $p_{i}$ norm, with $s_{i}, p_{i} \in \mathbb{N}$, such that every finite truncation of $u_{i}$ is in $\ell_{p_{i}}^{s_{i}}$. More explicitly, let $u_{i}(k)$ denote the $k^{\text {th }}$ element of $u_{i}$ and define

$$
P_{T} u_{i}= \begin{cases}u_{i}(k) & \text { for } k \leq T \\ 0 & \text { otherwise }\end{cases}
$$

Then we say $u_{i} \in \tilde{\ell}_{p_{i}}^{s_{i}}$ if and only if $P_{T} u_{i}$ has finite $p_{i}$-norm for all values of $T$. Using this definition, the full input space to the system is

$$
\tilde{\ell}_{p}^{s}=\prod_{i=1}^{N} \tilde{\ell}_{p_{i}}^{s_{i}}
$$

where the product is meant in the Cartesian sense, and the system produces outputs in

$$
y \in \tilde{\ell}_{q}^{r}
$$


In this paper we consider the cases where $p_{i}=1$ for all $i \in I$ or $p_{i}=2$ for all $i \in I$. In the case of $p_{i}=1$, the full input space to the system is $\tilde{\ell}_{1}^{s}$ and we use the ordinary 1-norm on this space. For $p_{i}=2$, we likewise use the ordinary 2-norm on $\tilde{\ell}_{2}^{s}$. While each of $\|\cdot\|_{1}$ and $\|\cdot\|_{2}$ will be used for both the 1 -norm and 2 -norm on $\mathbb{R}^{n}$ and $\tilde{\ell}_{p}^{s}$, the intent of each symbol can be discerned from its argument each time it is used.

To implement differential privacy, we must specify which inputs we wish to generate "similar" outputs. To do this, fix a real number $B>0$ and define the binary symmetric adjacency relation $\operatorname{Adj}_{B}: \tilde{\ell}_{p}^{s} \times \tilde{\ell}_{p}^{s} \rightarrow\{0,1\}$ as

$$
\operatorname{Adj}_{B}(u, \tilde{u})=1 \Leftrightarrow\|u-\tilde{u}\|_{p} \leq B
$$

Two inputs $u$ and $\tilde{u}$ for which $\operatorname{Adj}(u, \tilde{u})=1$ are called "adjacent."

Towards making precise the notion of "similar" outputs, fix a probability space $(\Omega, \mathcal{F}, \mathbb{P})$ and let $\mathscr{B}^{d}$ denote the Borel $\sigma$-algebra on $\mathbb{R}^{d}$. Differential privacy is enforced by a mechanism, which is a map $M$ taking the form

$$
M: \tilde{\ell}_{p}^{s} \times \Omega \rightarrow \tilde{\ell}_{q}^{r}
$$

and the role of a mechanism is to approximate a system whose inputs are sensitive information. We now state the definition of a differentially private mechanism. In this definition, we use a $\sigma$-algebra over $\tilde{\ell}_{q}^{r}$, denoted $\Sigma_{q, r} \cdot 1$

Definition 1: A mechanism $M: \tilde{\ell}_{p}^{s} \times \Omega \rightarrow \tilde{\ell}_{q}^{r}$ is $(\epsilon, \delta)$-differentially private if and only if, for all adjacent $u, \tilde{u} \in \tilde{\ell}_{p}^{s}$ we have

$$
\mathbb{P}(M(u) \in S) \leq e^{\epsilon} \mathbb{P}(M(\tilde{u}) \in S)+\delta
$$

for all $S \in \Sigma_{q, r}$.

In Equation (5) it is $\epsilon$ and $\delta$ that determine the privacy policy and smaller values of each imply a greater level of privacy for users. In general $\epsilon$ should be kept small and typical values

\footnotetext{
${ }^{1}$ An explicit construction of this $\sigma$-algebra can be found in [21 Section III-A], though we avoid a lengthy exposition on $\Sigma_{q, r}$ due to the relatively minor role its technical details play in the current work.
} 
for $\epsilon$ range from 0.1 to $\ln 3$. On the other hand, $\delta$ should be kept as small as possible because it allows for zero probability events for $M(\tilde{u})$ to have non-zero probability for $M(u)$ and therefore can allow for important losses in privacy by making it easy for an adversary to distinguish between outputs. Common values for $\delta$ range from 0 to $0.05 ;(\epsilon, 0)$-differential privacy is called $\epsilon$-differential privacy and, in general, $\epsilon$-differential privacy is stronger than $(\epsilon, \delta)$-differential privacy precisely because of the aforementioned losses in privacy that can come from $\delta>0$. For this reason, $(\epsilon, \delta)$-differential privacy can be regarded as a $\delta$-approximate form of $\epsilon$-differential privacy [38]. For a fixed value of $\epsilon$, the benefit of using even small values of $\delta>0$ is that the variance of noise added can be reduced while maintaining "almost" the same level of privacy.

\section{B. Private Optimization Problem Statement}

In the setting of Problem 0.2 , we want to protect the state trajectory, $\boldsymbol{x}=(x(k))_{k \in \mathbb{N}}$, which is a sensitive signal in $\tilde{\ell}_{p}^{s}$, and in so doing we protect each individual agent's state trajectory; for

agent $i$ this is $\boldsymbol{x}_{i} \in \tilde{\ell}_{p_{i}}^{s_{i}}$. As discussed in Section II], keeping individual agents' state trajectories private is necessary when the cloud computes $g_{x_{i}}$ and $\mu$ at each time $k$. To implement privacy in these computations, we regard each $g_{x_{i}}$ as a deterministic, causal, memory-less dynamical system and seek to make each such system differentially private. Similarly, we regard $g$ as a deterministic, causal, memory-less dynamical system and seek to make it differentially private as well. Due to the post-processing property of differential privacy, computing $\mu$ using a private form of $g$ also implies that $\mu$ keeps each $\boldsymbol{x}_{i}$ private.

As discussed in Section II] the agents do not communicate with each other at all and, instead, each agent only sends its state to the cloud. The cloud handles all required centralized computations and sends (privatized forms of) their results to the agents. Denoting by $\hat{g}_{x_{i}}$ and $\hat{g}$ the private forms of $g_{x_{i}}$ and $g$, respectively, at time $k$ the cloud sends to agent $i$ the vector

$$
\hat{p}_{i}(k)=\hat{g}_{x_{i}}(x(k))^{T} \hat{g}(x(k)) .
$$

We are interested in having a team of agents optimize by having the cloud send agent $i$ only $\hat{p}_{i}(k)$ at time $k$. We require that $\hat{p}_{i}(k)$ protect $\boldsymbol{x}_{i}$ for all $i \in I$, and we implement privacy by 
approximating $g_{x_{i}}$ (for all $i \in I$ ) and $g$ by differentially private mechanisms. Using this method of communications, we state the following problem that incorporates both optimization and privacy objectives, and respects the fact that the objectives and constraints in this problem are sensitive data.

Problem 1: (Private optimization) Solve Problem 0.2 using Algorithm 1 while

i. the agents communicate only with the cloud (i.e., there is no inter-agent communication)

ii. the cloud makes the systems $g$ and $g_{x_{i}}, i \in I$ (whose inputs are the agents' state trajectories) differentially private in the sense of Definition 1

iii. agent $i$ does not share $f_{i}$ or $X_{i}$ with any other agent or the cloud iv. the cloud does not share $g$ with any agent.

Towards solving Problem 1, we now review mechanisms which implement differential privacy for dynamical systems.

\section{Privacy-Preserving Mechanisms}

To define a mechanism for enforcing differential privacy, we must first also define the sensitivity of a system, which is used to determine the variance of noise that must be added in a privacy-preserving mechanism. Letting $\mathcal{G}$ be a deterministic causal system, the sensitivity of $\mathcal{G}$ is an upper bound on the distance between $\mathcal{G}(u)$ and $\mathcal{G}(\tilde{u})$ whenever $\operatorname{Adj}_{B}(u, \tilde{u})=1$ holds. Formally we define the $\ell_{p}$ sensitivity of $\mathcal{G}$, denoted $\Delta_{p} \mathcal{G}$, as

$$
\Delta_{p} \mathcal{G}:=\sup _{u, \tilde{u}: \operatorname{Adj}_{B}(u, \tilde{u})=1}\|\mathcal{G}(u)-\mathcal{G}(\tilde{u})\|_{p}
$$

The mechanism we will use for $\epsilon$-differential privacy is the Laplace mechanism, which adds noise drawn from a Laplace distribution. Below we use the notation $\operatorname{Lap}(\mu, b)$ to denote the Laplace distribution with mean $\mu$ and scale parameter $b$.

Theorem 1: ([21, Theorem 4]) Let the adjacency relation defined in Equation (4) be used with $p=1$ and let $\mathcal{G}$ be a system with sensitivity $\Delta_{1} \mathcal{G}$. Let a constant $\epsilon \geq 0$ be given and recall that 
$r$ is the dimension of the output space. Then the mechanism

$$
M(x)=\mathcal{G}(x)+w
$$

where $w(k) \sim \operatorname{Lap}(0, b / \epsilon)^{r}$ and $b \geq \Delta_{1} \mathcal{G}$ is $\epsilon$-differentially private.

For $(\epsilon, \delta)$-differential privacy, we will use the Gaussian mechanism. Its definition requires that we first define $\kappa(\delta, \epsilon)$ using the $\mathcal{Q}$-function,

$$
\mathcal{Q}(y):=\frac{1}{\sqrt{2 \pi}} \int_{y}^{\infty} e^{-\frac{v^{2}}{2}} d v
$$

The function $\kappa(\delta, \epsilon)$ is defined for $\epsilon \geq 0$ and $0<\delta<\frac{1}{2}$ as

$$
\kappa(\delta, \epsilon):=\frac{1}{2 \epsilon}\left(K_{\delta}+\sqrt{K_{\delta}^{2}+2 \epsilon}\right)
$$

where $K_{\delta}=\mathcal{Q}^{-1}(\delta)$. We now define the Gaussian mechanism.

Theorem 2: ([21, Theorem 3]) Let the adjacency relation defined in Equation (4) be used with $p=2$ and let $\mathcal{G}$ be a system with sensitivity $\Delta_{2} \mathcal{G}$, with constants $\epsilon \geq 0$ and $0<\delta<\frac{1}{2}$ given and $r$ the dimension of the output space. Then the mechanism

$$
M(x)=\mathcal{G}(x)+w
$$

where $w(k) \sim \mathcal{N}\left(0, \sigma^{2} I_{r}\right)$ is $(\epsilon, \delta)$-differentially private for $\sigma \geq \kappa(\delta, \epsilon) \Delta_{2} \mathcal{G}$.

Theorems 1 and 2 provide a lower bound on the variance of each noise that is added and we assume that these variances are also chosen to be finite. We now compute the sensitivities that are needed to implement differential privacy in Problem 1.

\section{Computing Sensitivities}

In Problem 1 it is desired to protect the value of $\boldsymbol{x}$, including from agents in the network. In the per-agent update law in Equation (3), $x(k)$ appears in $g_{x_{i}}$ and $g_{x_{i}}$ must therefore be made private before the cloud sends $g_{x_{i}}(x(k))$ to agent $i$. To protect $\boldsymbol{x}$ in this way, the cloud adds noise directly to $g_{x_{i}}(x(k))$, and the variance of noise that must be added depends on the 
sensitivity of $g_{x_{i}}$. To compute the sensitivity of $g_{x_{i}}$ we regard it as a memoryless dynamical system and generalize it to act on entire signals of states. Recalling that $x(k) \in X$ for all $k$, under Assumption 2, $X$ is bounded and therefore $x(k)$ is as well for all $k \in \mathbb{N}$. Then $\boldsymbol{x} \in \tilde{\ell}_{p}^{n}$.

We now overload the notation $g_{x_{i}}$ by allowing it to act on elements of $\tilde{\ell}_{p}^{n}$. In particular, $g_{x_{i}}$ acts on elements of $\mathbb{R}^{n}$ as before and for state trajectories $\boldsymbol{x} \in \tilde{\ell}_{p}^{n}$ we define

$$
g_{x_{i}}(\boldsymbol{x}):=\left(g_{x_{i}}(x(k))\right)_{k \in \mathbb{N}} \cdot
$$

We now fix a real scalar $B>0$. For two state trajectories, $\boldsymbol{x}, \tilde{\boldsymbol{x}} \in \tilde{\ell}_{p}^{n}$ such that $\operatorname{Adj}_{B}(\boldsymbol{x}, \tilde{\boldsymbol{x}})=1$ holds, we compute the sensitivity of $g_{x_{i}}$ according to

$$
\begin{aligned}
\Delta_{p} g_{x_{i}}=\sup _{\boldsymbol{x}, \tilde{\boldsymbol{x}}: \operatorname{Adj}_{B}(\boldsymbol{x}, \tilde{\boldsymbol{x}})=1}\left\|g_{x_{i}}(\boldsymbol{x})-g_{x_{i}}(\tilde{\boldsymbol{x}})\right\|_{p} & \\
& \leq \sup _{\boldsymbol{x}, \tilde{\boldsymbol{x}}: \operatorname{Adj}_{B}(\boldsymbol{x}, \tilde{\boldsymbol{x}})=1} K_{p}^{i} \sqrt[p]{\sum_{k=0}^{\infty}\|x(k)-\tilde{x}(k)\|_{p}^{p}} \leq K_{p}^{i} B,
\end{aligned}
$$

where we have used $\|\boldsymbol{x}-\tilde{\boldsymbol{x}}\|_{p} \leq B$ and where this bound on the sensitivity holds for $g_{x_{i}}$ for all $i \in I$.

In computing $\mu(k)$, the cloud must also add noise in some fashion because $\mu(k)$ depends upon $x(k)$. We regard $g$ as a dynamical system and make it private, and the resilience of differential privacy to post-processing guarantees that $\boldsymbol{\mu}=(\mu(k))_{k \in \mathbb{N}}$ keeps $\boldsymbol{x}$ private. To compute the sensitivity of $g$ we extend it to act on $\boldsymbol{x} \in \tilde{\ell}_{p}^{n}$ as above. For $\boldsymbol{x}, \tilde{\boldsymbol{x}} \in \tilde{\ell}_{p}^{n} \operatorname{satisfying}_{\operatorname{Adj}_{B}}(\boldsymbol{x}, \tilde{\boldsymbol{x}})=1$ we use the same procedure as was used above for $g_{x_{i}}$ to find

$$
\Delta_{p} g=\sup _{\boldsymbol{x}, \tilde{\boldsymbol{x}}: \operatorname{Adj}_{B}(\boldsymbol{x}, \tilde{\boldsymbol{x}})=1}\|g(\boldsymbol{x})-g(\tilde{\boldsymbol{x}})\|_{p} \leq K_{p}^{g} B .
$$

Having computed the requisite sensitivities, we return to solving Problem 1.

\section{E. Optimizing in the Presence of Noise}

We now examine how noise appears in Algorithm 1 once it has been added for privacy. For $g_{x_{i}}(x(k))$ we add noise $w_{i}(k) \in \mathbb{R}^{m \times n_{i}}$ drawn from either a Laplace or Gaussian distribution 
and for $g(x(k))$ we add noise $w_{g}(k) \in \mathbb{R}^{m}$ drawn from the same class of distribution as the $w_{i}$, with all noises independent. Define $w_{x}$ by

$$
w_{x}=\left(w_{1} w_{2} \cdots w_{n}\right) \in \mathbb{R}^{m \times n}
$$

In ensemble form the private dynamics under consideration are

$$
\begin{aligned}
z(k+1) & =\left(\begin{array}{c}
x(k+1) \\
\mu(k+1)
\end{array}\right) \\
& =\Pi_{Z}\left[z(k)-\gamma_{k}\left(\begin{array}{c}
f_{x}(x(k))+\left(\frac{\partial g}{\partial x}(x(k))+w_{x}(k)\right)^{T} \mu(k)+\alpha_{k} x(k) \\
-g(x(k))+w_{g}(k)+\alpha_{k} \mu(k)
\end{array}\right)\right] .
\end{aligned}
$$

Expanding, we find

$$
\begin{aligned}
z(k+1) & =\left(\begin{array}{c}
x(k+1) \\
\mu(k+1)
\end{array}\right) \\
& =\Pi_{Z}\left[z(k)-\gamma_{k}\left(\begin{array}{c}
f_{x}(x(k))+\frac{\partial g}{\partial x}(x(k))^{T} \mu(k)+w_{x}(k)^{T} \mu(k)+\alpha_{k} x(k) \\
-g(x(k))+w_{g}(k)+\alpha_{k} \mu(k)
\end{array}\right)\right] .
\end{aligned}
$$

Because $\mu(k) \in \mathbb{R}_{+}^{m}$, each element of $w_{x}(k)^{T} \mu(k)$ is some weighted combination of elements of $w_{x}(k)$ with non-negative weights. Combined with the independence of the noises used for privacy, this results in each entry of $w_{x}(k)^{T} \mu(k)$ being a random variable having variance that is the weighted sum of variances of elements of $w_{x}(k)$. With this in mind we define the random vector $w_{s}(k)=w_{x}(k)^{T} \mu(k)$ (which we note has finite variance since $\mu(k)$ is contained in $\mathbb{M}$ and $w_{x}(k)$ has finite variance), and zero mean (because $w_{i}(k)$ has zero mean for all $k \in \mathbb{N}$ and all $i \in I$ ). Then we can rewrite Equation (6) as

$$
\begin{aligned}
z(k+1) & =\Pi_{Z}\left[z(k)-\gamma_{k}\left(\begin{array}{c}
f_{x}(x(k))+\frac{\partial g}{\partial x}(x(k))^{T} \mu(k)+w_{s}(k)+\alpha_{k} x(k) \\
-g(x(k))+w_{g}(k)+\alpha_{k} \mu(k)
\end{array}\right)\right] \\
& =\Pi_{Z}\left[z(k)-\gamma_{k}\left(G(z(k))+\alpha_{k} z(k)+w(k)\right)\right]
\end{aligned}
$$

where $w(k)$ denotes the noise added at timestep $k$ and aggregates all noisy signals used for 
privacy. We state this stochastic update law as Algorithm 2 .

Algorithm 2: Given $z(0) \in Z$, apply the update law

$$
z(k+1)=\Pi_{Z}\left[z(k)-\gamma_{k}\left(G(z(k))+\alpha_{k} z(k)+w(k)\right)\right]
$$

until a fixed point $\hat{z} \in Z$ is reached.

We note that by its definition $\mathbb{E}[w(k)]=0$, and observe that this noisy update law is equivalent to Algorithm 1 with an additional noise term added.

\section{Convergence of Private Optimization}

In this section we prove the convergence of Algorithm 2, Algorithm 2 was first presented in [34] without noise and was presented in its noisy form in [35]. Both papers omit proofs and, due to the heavy dependence of this work upon Algorithm 2 we provide a proof here. To the best of our knowledge a proof of the convergence of Algorithm 2] as stated in [35] is not available in the literature; similar work is presented in [39], [40] which cover algorithms related to Algorithm 2, though those works impose additional assumptions upon $\alpha_{k}$ and $\gamma_{k}$ due to the differences in the problems studied in those works.

\section{A. Main Convergence Result}

Now we explore in depth solving variational inequalities using a Tikhonov regularized projection method, the basic elements of which are covered in [33, Section 12.2]. Earlier it was stated that if $\operatorname{SOL}(K, F) \neq \emptyset$, then for $\xi_{k} \in S O L\left(K, F+\alpha_{k} I\right)$ we have $\xi_{k} \rightarrow z_{0}$ where $z_{0}$ is the least-norm element of $\operatorname{SOL}(K, F)$. Using that $\left\{\xi_{k}\right\}_{k \in \mathbb{N}}$ is a convergent sequence, we find that $\left\{\left\|\xi_{k}\right\|\right\}_{k \in \mathbb{N}}$ is bounded and, in particular, there is some $M_{\xi}$ such that $\left\|\xi_{k}\right\| \leq M_{\xi}$ for all $k$, e.g., $\left\|\xi_{k}\right\| \leq \sup _{z \in Z}\|z\|$.

Using this fact, the following lemma relates points $z(k)$ generated by Algorithm 2 to successive solutions to the problems $V I\left(Z, G+\alpha_{k} I\right)$ (each with $\alpha_{k}$ held constant). Recalling that $\xi_{k}$ is the unique solution to $\operatorname{VI}\left(Z, G+\alpha_{k} I\right)$, we have the following result. 
Lemma 1: For all $k \in \mathbb{N}$

$$
\left\|z(k)-\xi_{k}\right\|^{2} \leq\left(1+\gamma_{k} \alpha_{k}\right)\left\|z(k)-\xi_{k-1}\right\|^{2}+M_{\xi}^{2}\left(\frac{\alpha_{k-1}-\alpha_{k}}{\alpha_{k}}\right)^{2}\left(\frac{1+\gamma_{k} \alpha_{k}}{\gamma_{k} \alpha_{k}}\right) .
$$

Proof: First note that because $\xi_{k}$ solves $V I\left(K, G+\alpha_{k} I\right)$, we have

$$
\left(\xi_{k-1}-\xi_{k}\right)^{T}\left(G\left(\xi_{k}\right)+\alpha_{k} \xi_{k}\right) \geq 0 .
$$

Similarly for $\xi_{k-1}$ we find

$$
\left(\xi_{k}-\xi_{k-1}\right)^{T}\left(G\left(\xi_{k-1}\right)+\alpha_{k-1} \xi_{k-1}\right) \geq 0 .
$$

Summing Equations (7) and (8), and using the monotonicity of $G$ gives

$$
\left(\xi_{k-1}-\xi_{k}\right)^{T}\left(\alpha_{k} \xi_{k}-\alpha_{k-1} \xi_{k-1}\right) \geq 0 .
$$

Adding and subtracting $\alpha_{k} \xi_{k-1}$ inside the second set of parentheses then gives

$$
\left(\xi_{k-1}-\xi_{k}\right)^{T}\left(\alpha_{k} I-\alpha_{k-1} I\right) \xi_{k-1}
$$

$$
\geq \alpha_{k}\left(\xi_{k-1}-\xi_{k}\right)^{T}\left(\xi_{k-1}-\xi_{k}\right)=\alpha_{k}\left\|\xi_{k-1}-\xi_{k}\right\|^{2} .
$$

Using the Cauchy-Schwarz inequality results in

$$
\left\|\xi_{k-1}-\xi_{k}\right\| \leq \frac{\left|\alpha_{k-1}-\alpha_{k}\right|}{\alpha_{k}} M_{\xi}
$$

Expanding the term $\left\|z(k)+\xi_{k-1}-\xi_{k-1}-\xi_{k}\right\|^{2}$ and applying Equation (9) then gives

$$
\begin{aligned}
\left\|z(k)-\xi_{k}\right\|^{2} \leq\left\|z(k)-\xi_{k-1}\right\|^{2}+\left(\frac{\alpha_{k-1}-\alpha_{k}}{\alpha_{k}}\right)^{2} & M_{\xi}^{2} \\
& +2\left(\frac{\left|\alpha_{k-1}-\alpha_{k}\right|}{\alpha_{k}}\right) M_{\xi}\left\|z(k)-\xi_{k-1}\right\| .
\end{aligned}
$$


For the third term on the right-hand side above we have

$$
\begin{aligned}
2\left(\frac{\left|\alpha_{k-1}-\alpha_{k}\right|}{\alpha_{k}}\right) M_{\xi} \| z(k) & -\xi_{k-1} \| \\
=2 \sqrt{\gamma_{k} \alpha_{k}} \| z(k) & -\xi_{k-1} \|\left(\frac{\left|\alpha_{k-1}-\alpha_{k}\right|}{\alpha_{k} \sqrt{\gamma_{k} \alpha_{k}}}\right) M_{\xi} \\
& \leq \gamma_{k} \alpha_{k}\left\|z(k)-\xi_{k-1}\right\|^{2}+M_{\xi}^{2}\left(\frac{\left(\alpha_{k-1}-\alpha_{k}\right)^{2}}{\gamma_{k} \alpha_{k}^{3}}\right),
\end{aligned}
$$

where we have used that fact that $a^{2}+b^{2} \geq 2 a b$ for $a, b \in \mathbb{R}$.

Substituting Equation (11) into Equation (10) gives the desired result.

The other lemma we need concerns the convergence of sequences of random variables and enables a Lyapunov-like argument to be made for their convergence.

Lemma 2: ([41], Lemma 10, Page 49) Let $v_{0}, \ldots, v_{k}$ be a sequence of independent random variables with $v_{k} \geq 0$ and $\mathbb{E}\left[v_{0}\right]<\infty$. Suppose that

$$
\mathbb{E}\left[v_{k+1}\right] \leq\left(1-\tau_{k}\right) v_{k}+\sigma_{k}
$$

with

$$
0 \leq \tau_{k} \leq 1, \quad \sigma_{k} \geq 0, \quad \sum_{k=0}^{\infty} \tau_{k}=\infty, \quad \frac{\sigma_{k}}{\tau_{k}} \rightarrow 0
$$

Then $\mathbb{E}\left[v_{k}\right] \rightarrow 0$. If, in addition, we have

$$
\sum_{k=0}^{\infty} \sigma_{k}<\infty
$$

then $v_{k} \rightarrow 0$ almost surely and

$$
\mathbb{P}\left(v_{j} \leq \epsilon \text { for all } j \geq k\right) \geq 1-\frac{1}{\epsilon}\left(\mathbb{E}\left[v_{k}\right]+\sum_{i=k}^{\infty} \sigma_{i}\right)
$$

We now prove the convergence of Algorithm 2.

Theorem 3: Let Assumptions 1-4 hold. Suppose that $\gamma_{k}>0$ and $\alpha_{k}>0$ satisfy the following four conditions: 
1) $\sum_{k=0}^{\infty} \gamma_{k} \alpha_{k}=\infty$

2) $\frac{\gamma_{k}}{\alpha_{k}} \rightarrow 0$

3) $\alpha_{k} \rightarrow 0$

4) $\frac{\left(\alpha_{k-1}-\alpha_{k}\right)}{\gamma_{k} \alpha_{k}^{2}} \rightarrow 0$.

Then for noise signal $w$ with $\mathbb{E}[w(k)]=0$ and bounded variance for all $k \in \mathbb{N}$, for the update rule

$$
z(k+1)=\Pi_{Z}\left[z(k)-\gamma_{k}\left(G(z(k))+\alpha_{k} z(k)+w(k)\right)\right]
$$

we have $\mathbb{E}\left[\left\|z(k)-z_{0}\right\|^{2}\right] \rightarrow 0$, where $z_{0}$ is the least-norm solution to Problem 0.2 .

Let $L_{G}$ be the Lipschitz constant of $G$. If, in addition to the above, the sequence of terms

$$
\begin{array}{r}
\sigma_{k}:=\left(1-\gamma_{k} \alpha_{k}\left(2-\gamma_{k} \alpha_{k}-\frac{\gamma_{k}}{\alpha_{k}} L_{G}^{2}-2 \gamma_{k} L_{G}\right)\right) M_{\xi}^{2} \cdot\left(\frac{\alpha_{k-1}-\alpha_{k}}{\alpha_{k}}\right)^{2}\left(\frac{1+\gamma_{k} \alpha_{k}}{\gamma_{k} \alpha_{k}}\right) \\
+\gamma_{k}^{2} \mathbb{E}\left[\|w(k)\|^{2}\right]
\end{array}
$$

is summable, then the convergence estimate

$$
\mathbb{P}\left(\left\|z(j)-\xi_{j-1}\right\|^{2} \leq \epsilon \text { for all } j \geq k\right) \geq 1-\frac{1}{\epsilon}\left(\mathbb{E}\left[\left\|z(k)-\xi_{k-1}\right\|^{2}\right]+\sum_{i=k}^{\infty} \sigma_{i}\right)
$$

holds.

Proof: It was established in Section II-A that $S O L(Z, G) \neq \emptyset$ so that $\xi_{k} \rightarrow z_{0}$ where $z_{0}$ is the least-norm element of $\operatorname{SOL}(Z, G)$ and where $\xi_{k}$ solves $V I\left(Z, G+\alpha_{k} I\right)$. We now show that $z(k+1) \rightarrow \xi_{k}$

Because $\xi_{k}$ solves $V I\left(Z, G+\alpha_{k} I\right)$ we have

$$
\xi_{k}=\Pi_{Z}\left[\xi_{k}-\gamma_{k}\left(G\left(\xi_{k}\right)+\alpha_{k} \xi_{k}\right)\right]
$$

Using the non-expansive property of the projection operator and taking the expectation of both 
sides we find

$$
\begin{aligned}
& \mathbb{E}\left[\left\|z(k+1)-\xi_{k}\right\|^{2}\right]= \\
& \mathbb{E}\left[\left\|\Pi_{Z}\left[z(k)-\gamma_{k}\left(G(z(k))+\alpha_{k} z(k)+w(k)\right)\right]-\Pi_{Z}\left[\xi_{k}-\gamma_{k}\left(G\left(\xi_{k}\right)+\alpha_{k} \xi_{k}\right)\right]\right\|^{2}\right] \\
& \leq \mathbb{E}\left[\left\|z(k)-\xi_{k}+\gamma_{k}\left(G\left(\xi_{k}\right)-G(z(k))\right)-\gamma_{k} \alpha_{k}\left(z(k)-\xi_{k}\right)-\gamma_{k} w(k)\right\|^{2}\right] \\
& \leq\left\|z(k)-\xi_{k}\right\|^{2}-2 \gamma_{k} \alpha_{k}\left\|z(k)-\xi_{k}\right\|^{2}+\gamma_{k}^{2}\left\|G\left(\xi_{k}\right)-G(z(k))\right\|^{2} \\
& +2 \gamma_{k}^{2} \alpha_{k}\left(G\left(\xi_{k}\right)-G(z(k))\right)^{T}\left(\xi_{k}-z(k)\right)+\gamma_{k}^{2} \alpha_{k}^{2}\left\|\xi_{k}-z(k)\right\|^{2}+\gamma_{k}^{2} \mathbb{E}\left[\|w(k)\|^{2}\right]
\end{aligned}
$$

where the last inequality follows from the monotonicity of $G$, and where the fact that $\mathbb{E}[w(k)]=0$ has caused all terms containing $w(k)$ except $\mathbb{E}\left[\|w(k)\|^{2}\right]$ to vanish.

Using the Cauchy-Schwarz inequality then gives

$$
\begin{aligned}
\mathbb{E}[\| z(k+1)- & \left.\xi_{k} \|^{2}\right] \leq\left\|z(k)-\xi_{k}\right\|^{2}-2 \gamma_{k} \alpha_{k}\left\|z(k)-\xi_{k}\right\|^{2} \\
+ & +\gamma_{k}^{2}\left\|G\left(\xi_{k}\right)-G(z(k))\right\|^{2}+2 \gamma_{k}^{2} \alpha_{k}\left\|G\left(\xi_{k}\right)-G(z(k))\right\|\left\|\xi_{k}-z(k)\right\| \\
& +\gamma_{k}^{2} \alpha_{k}^{2}\left\|\xi_{k}-z(k)\right\|^{2}+\gamma_{k}^{2} \mathbb{E}\left[\|w(k)\|^{2}\right] .
\end{aligned}
$$

Assumptions 1,3 and the compactness of $\mathbb{M}$ together imply that $G$ is Lipschitz and, denoting its Lipschitz constant by $L_{G}$, we have

$$
\begin{aligned}
& \mathbb{E}\left[\left\|z(k+1)-\xi_{k}\right\|^{2}\right] \leq \\
&\left(1-2 \gamma_{k} \alpha_{k}+\gamma_{k}^{2} L_{G}^{2}+2 \gamma_{k}^{2} \alpha_{k} L_{G}+\gamma_{k}^{2} \alpha_{k}^{2}\right)\left\|z(k)-\xi_{k}\right\|^{2}+\gamma_{k}^{2} \mathbb{E}\left[\|w(k)\|^{2}\right] .
\end{aligned}
$$

Defining

$$
\theta_{k}:=1-\gamma_{k} \alpha_{k}\left(2-\gamma_{k} \alpha_{k}-\frac{\gamma_{k}}{\alpha_{k}} L_{G}^{2}-2 \gamma_{k} L_{G}\right)
$$

and

$$
\rho_{k}:=M_{\xi}^{2}\left(\frac{\alpha_{k-1}-\alpha_{k}}{\alpha_{k}}\right)^{2}\left(\frac{1+\gamma_{k} \alpha_{k}}{\gamma_{k} \alpha_{k}}\right)
$$


applying Lemma 1 then gives

$$
\mathbb{E}\left[\left\|z(k+1)-\xi_{k}\right\|^{2}\right] \leq \theta_{k}\left(1+\gamma_{k} \alpha_{k}\right)\left\|z(k)-\xi_{k-1}\right\|^{2}+\theta_{k} \rho_{k}+\gamma_{k}^{2} \mathbb{E}\left[\|w(k)\|^{2}\right] .
$$

By hypothesis we have

$$
\gamma_{k} \alpha_{k} \rightarrow 0, \quad \frac{\gamma_{k}}{\alpha_{k}} \rightarrow 0, \quad \gamma_{k} \rightarrow 0
$$

with $\alpha_{k}>0$ and $\gamma_{k}>0$ for all $k$. Then there exists an $M>0$ such that for all $k \geq M$ we have

$$
\gamma_{k} \alpha_{k} \in(0,1) \text { and } 0 \leq 1-2 \gamma_{k} \alpha_{k} \leq \theta_{k} \leq 1-\gamma_{k} \alpha_{k}
$$

Then for all $k \geq M$

$$
\theta_{k}\left(1+\gamma_{k} \alpha_{k}\right) \leq \theta_{k}+\gamma_{k} \alpha_{k}
$$

and thus for all $k \geq M$

$$
\theta_{k}\left(1+\gamma_{k} \alpha_{k}\right) \leq 1-\gamma_{k} \alpha_{k}\left(1-\gamma_{k} \alpha_{k}-\frac{\gamma_{k}}{\alpha_{k}} L_{G}^{2}-2 \gamma_{k} L_{G}\right) \in(0,1)
$$

In particular, take some $\theta \in(0,1)$ so that

$$
1-\gamma_{k} \alpha_{k}\left(1-\gamma_{k} \alpha_{k}-\frac{\gamma_{k}}{\alpha_{k}} L_{G}^{2}-2 \gamma_{k} L_{G}\right) \leq 1-\gamma_{k} \alpha_{k} \theta
$$

for all $k \geq M$. Then $1-\gamma_{k} \alpha_{k} \theta \in(0,1)$. Setting $\tau_{k}=\gamma_{k} \alpha_{k} \theta$ and $\sigma_{k}=\rho_{k} \theta_{k}+\gamma_{k}^{2} \mathbb{E}\left[\|w(k)\|^{2}\right]$ we rewrite Equation (13) as

$$
\mathbb{E}\left[\left\|z(k+1)-\xi_{k}\right\|^{2}\right] \leq\left(1-\tau_{k}\right)\left\|z(k)-\xi_{k-1}\right\|^{2}+\sigma_{k}
$$

All that remains is to show that the conditions of Lemma 2 are met. First, $\tau_{k} \in(0,1)$ by construction. For all $k \geq M$ we have $\rho_{k} \geq 0$ and $\theta_{k} \geq 0$ so that $\sigma_{k} \geq 0$. Regarding summability of $\tau_{k}$ we find

$$
\sum_{k=M}^{\infty} \tau_{k}=\theta \sum_{k=M}^{\infty} \gamma_{k} \alpha_{k}=\infty
$$


by hypothesis. To show that $\sigma_{k} / \tau_{k} \rightarrow 0$ we have

$$
\begin{aligned}
\frac{\sigma_{k}}{\tau_{k}} & =\frac{\theta_{k}\left(\frac{\alpha_{k-1}-\alpha_{k}}{\alpha_{k}}\right)^{2}\left(\frac{1+\gamma_{k} \alpha_{k}}{\gamma_{k} \alpha_{k}}\right) M_{\xi}^{2}+\gamma_{k}^{2} \mathbb{E}\left[\|w(k)\|^{2}\right]}{\gamma_{k} \alpha_{k} \theta} \\
& =\left(1-2 \gamma_{k} \alpha_{k}+\gamma_{k}^{2} \alpha_{k}^{2}+\gamma_{k}^{2} L_{G}^{2}+2 \alpha_{k} \gamma_{k}^{2} L_{G}\right) M_{\xi}^{2} \\
& \cdot\left(\frac{\alpha_{k-1}-\alpha_{k}}{\gamma_{k} \alpha_{k}^{2}}\right)^{2}\left(1+\gamma_{k} \alpha_{k}\right) \frac{1}{\theta}+\frac{1}{\theta} \frac{\gamma_{k}}{\alpha_{k}} \mathbb{E}\left[\|w(k)\|^{2}\right]
\end{aligned}
$$

Using the hypotheses regarding $\gamma_{k}$ and $\alpha_{k}$ we have

$$
1-2 \gamma_{k} \alpha_{k}+\gamma_{k}^{2} \alpha_{k}^{2}+\gamma_{k}^{2} L_{G}^{2}+2 \alpha_{k} \gamma_{k}^{2} L_{G} \rightarrow 1, \quad 1+\gamma_{k} \alpha_{k} \rightarrow 1
$$

along with

$$
\left(\frac{\alpha_{k-1}-\alpha_{k}}{\gamma_{k} \alpha_{k}^{2}}\right)^{2} \rightarrow 0
$$

so that the first term in $\frac{\sigma_{k}}{\tau_{k}}$ goes to zero. It was established in Section III-E that $\mathbb{E}\left[\|w(k)\|^{2}\right]$ is bounded above for all $k$, namely that $\mathbb{E}\left[\|w(k)\|^{2}\right] \leq K_{w}$ for some $K_{w}>0$. Because $\frac{\gamma_{k}}{\alpha_{k}} \rightarrow 0$ we have $\frac{\gamma_{k}}{\alpha_{k}} K_{w} \rightarrow 0$ and hence $\frac{\sigma_{k}}{\tau_{k}} \rightarrow 0$ as desired and the first part of the theorem follows from Lemma 2 .

When the sequence $\left\{\sigma_{k}\right\}_{k \in \mathbb{N}}$ is summable, the additional convergence estimate is a straightforward application of Lemma 2 as well.

One valid choice of $\gamma_{k}$ and $\alpha_{k}$ satisfying conditions 1-4 in Theorem 3 is

$$
\alpha=\bar{\alpha} k^{-c_{1}} \text { and } \gamma=\bar{\gamma} k^{-c_{2}}
$$

with $\bar{\alpha}>0, \bar{\gamma}>0,0<c_{1}<c_{2}$, and $c_{1}+c_{2}<1[35]$.

\section{B. Convergence Rate Estimates}

For the above choice of step-size, we derive bounds on $c_{1}$ and $c_{2}$ which are sufficient to make $\sigma_{k}$ summable. As shown in the proof of Theorem 3, there exists an $M>0$ such that for all 
$k \geq M$ we have $\theta_{k} \in(0,1)$, so that for all $k \geq M$ we have

$$
\sigma_{k} \leq M_{\xi}^{2}\left(\frac{\alpha_{k-1}-\alpha_{k}}{\alpha_{k}}\right)^{2}\left(\frac{1+\gamma_{k} \alpha_{k}}{\gamma_{k} \alpha_{k}}\right)+\gamma_{k}^{2} \mathbb{E}\left[\|w(k)\|^{2}\right]
$$

To make the second term in Equation (15) summable, we can set

$$
\gamma_{k}=\bar{\gamma} k^{-c_{2}}
$$

with $c_{2}>\frac{1}{2}$. Again using $K_{w}$ to denote an upper bound on the variance of $w(k)$ gives

$$
\sum_{k=1}^{\infty} \gamma_{k}^{2} \mathbb{E}\left[\|w(k)\|^{2}\right] \leq \bar{\gamma}^{2} K_{w} \zeta\left(2 c_{2}\right)
$$

where $\zeta(\cdot)$ is the Riemann zeta function [42], defined as

$$
\zeta(p)=\sum_{n=1}^{\infty} \frac{1}{n^{p}}
$$

which takes finite values for arguments $p>1$.

Regarding the first term in Equation (15), we note that there is some $\hat{M}>0$ such that

$$
1 \leq \frac{1}{\bar{\alpha} \bar{\gamma} k^{-c_{1}} k^{-c_{2}}}
$$

for all $k \geq \hat{M}$ and therefore

$$
\left(\frac{\alpha_{k-1}-\alpha_{k}}{\alpha_{k}}\right)^{2}\left(\frac{1+\gamma_{k} \alpha_{k}}{\gamma_{k} \alpha_{k}}\right) \leq 2\left(\frac{\alpha_{k-1}-\alpha_{k}}{\alpha_{k}}\right)^{2} \frac{1}{\bar{\alpha} \bar{\gamma} k^{-\left(c_{1}+c_{2}\right)}}
$$

for all such $k$. Substituting $\alpha_{k}=\bar{\alpha} k^{-c_{1}}$ and expanding the squared term gives

$$
2\left(\frac{\alpha_{k-1}-\alpha_{k}}{\alpha_{k}}\right)^{2} \frac{1}{\bar{\alpha} \bar{\gamma} k^{-\left(c_{1}+c_{2}\right)}}=2 \frac{\left[\left(1-\frac{1}{k}\right)^{-2 c_{1}}-2\left(1-\frac{1}{k}\right)^{-c_{1}}+1\right]}{\bar{\alpha} \bar{\gamma} k^{-\left(c_{1}+c_{2}\right)}} .
$$

To approximate the terms containing $1 / k$ we use a (truncated) power series expansion, namely that for $x \in(-1,1)$

$$
(1-x)^{-r} \approx 1+r x+\frac{1}{2} r(r+1) x^{2}+\frac{1}{6} r(r+1)(r+2) x^{3} .
$$


Applying Equation (18) to Equation (17) gives

$$
2 \frac{\left[\left(1-\frac{1}{k}\right)^{-2 c_{1}}-2\left(1-\frac{1}{k}\right)^{-c_{1}}+1\right]}{\bar{\alpha} \bar{\gamma} k^{-\left(c_{1}+c_{2}\right)}} \approx \frac{2 c_{1}^{2}}{\bar{\alpha} \bar{\gamma} k^{2-\left(c_{1}+c_{2}\right)}}+\frac{2\left(c_{1}^{3}+c_{1}^{2}\right)}{\bar{\alpha} \bar{\gamma} k^{3-\left(c_{1}+c_{2}\right)}} .
$$

We see that sums of such terms are given by

$$
\begin{aligned}
& \sum_{k=1}^{\infty} 2 \frac{\left[\left(1-\frac{1}{k}\right)^{-2 c_{1}}-2\left(1-\frac{1}{k}\right)^{-c_{1}}+1\right]}{\bar{\alpha} \bar{\gamma} k^{-\left(c_{1}+c_{2}\right)}} \\
& \quad \approx \frac{2 c_{1}^{2}}{\bar{\alpha} \bar{\gamma}} \zeta\left(2-\left(c_{1}+c_{2}\right)\right)+\frac{2\left(c_{1}^{3}+c_{1}^{2}\right)}{\bar{\alpha} \bar{\gamma}} \zeta\left(3-\left(c_{1}+c_{2}\right)\right) .
\end{aligned}
$$

Returning to Equation (15) and using the results of Equations (16) and (19) gives

$$
\sum_{k=1}^{\infty} \sigma_{k} \lesssim \bar{\gamma}^{2} K_{w} \zeta\left(2 c_{2}\right)+\frac{2 c_{1}^{2}}{\bar{\alpha} \bar{\gamma}} \zeta\left(2-\left(c_{1}+c_{2}\right)\right)+\frac{2\left(c_{1}^{3}+c_{1}^{2}\right)}{\bar{\alpha} \bar{\gamma}} \zeta\left(3-\left(c_{1}+c_{2}\right)\right) .
$$

Due to the approximations made and ranges of $k$ considered in bounding this sum, we can only guarantee that the convergence estimate relying on $\sum_{k=1}^{\infty} \sigma_{k}$ will hold for $k \geq \max \{M, \hat{M}\}$. However, for $k \leq M$ we will often have $\sigma_{k}<0$ (as when $L_{G}$ is large) and thus we expect the bound in Equation (20) to hold for a range of values of $k \leq M$ because negative terms with such indices have been over-estimated by including positive terms at such indices in Equation 201. In addition, we expect $\bar{\alpha}$ and $\bar{\gamma}$ to be small enough that $\hat{M}$ will often be small, e.g., less than 10, thus allowing this bound to hold over a wide range of values of $k$.

To apply the bound in Equation (12), we also need to estimate the term $\mathbb{E}\left[\left\|z(k)-\xi_{k-1}\right\|^{2}\right]$. Returning to Equation (14) and taking the expectation of both sides one timestep earlier gives

$$
\mathbb{E}\left[\left\|z(k)-\xi_{k-1}\right\|^{2}\right] \leq\left(1-\tau_{k-1}\right) \mathbb{E}\left[\left\|z(k-1)-\xi_{k-2}\right\|^{2}\right]+\sigma_{k-1},
$$

which is a (time-varying) affine recurrence relation in the expected error in the optimization algorithm. Solving Equation (21) (see e.g., [43], Section 2.1.1.2), we find that

$$
\mathbb{E}\left[\left\|z(k)-\xi_{k-1}\right\|^{2}\right]=\left(\prod_{n=0}^{k-1}\left(1-\tau_{n}\right)\right)\left(\mathbb{E}\left[\left\|z(1)-\xi_{0}\right\|^{2}\right]+\sum_{m=0}^{k-1} \frac{\sigma_{m}}{\prod_{i=0}^{m}\left(1-\tau_{i}\right)}\right)
$$


Defining the diameter of the set $Z$ via $D_{z}=\sup _{z_{1}, z_{2} \in Z}\left\|z_{1}-z_{2}\right\|$, we can bound the initial error via $\mathbb{E}\left[\left\|z(1)-\xi_{0}\right\|^{2}\right] \leq D_{z}^{2}$, giving

$$
\mathbb{E}\left[\left\|z(k)-\xi_{k-1}\right\|^{2}\right] \leq\left(\prod_{n=0}^{k-1}\left(1-\tau_{n}\right)\right)\left(D_{z}^{2}+\sum_{m=0}^{k-1} \frac{\sigma_{m}}{\prod_{i=0}^{m}\left(1-\tau_{i}\right)}\right)
$$

In Equation (12), one can compute the sum $\sum_{i=k}^{\infty} \sigma_{i}$ by using the analytic bound for $\sum_{k=1}^{\infty} \sigma_{k}$ given in Equation (20) and subtracting the first $k-1$ values of $\sigma_{i}$ from this value. Combined with the upper bound on $\mathbb{E}\left[\left\|z(k)-\xi_{k-1}\right\|^{2}\right]$ given in Equation (22), one can then use the bound in Equation (12) to determine the probability with which the error in the optimization algorithm stays within some bound for all time. Having explored convergence in the presence of privacy, we now examine the trade-off between the two competing objectives of privacy and convergence.

\section{The Trade-off Between Privacy and Convergence}

In this section we derive a quantifiable trade-off between privacy and convergence, and for concreteness we focus on the case of $\epsilon$-differential privacy, though a similar trade-off can be derived for $(\epsilon, \delta)$-differential privacy.

Returning to Equation (13) we find the inequality

$$
\mathbb{E}\left[\left\|z(k+1)-\xi_{k}\right\|^{2}\right] \leq \theta_{k}\left(1+\alpha_{k} \gamma_{k}\right)\left\|z(k)-\xi_{k-1}\right\|^{2}+\theta_{k} \rho_{k}+\gamma_{k}^{2} \mathbb{E}\left[\|w(k)\|^{2}\right],
$$

where we see that only the term $\mathbb{E}\left[\|w(k)\|^{2}\right]$ depends upon the noise added for privacy. Given that $w(k)$ has zero mean, we find $\mathbb{E}\left[\|w(k)\|^{2}\right]=\operatorname{var}(w(k))$. In the case of $\epsilon$-differential privacy we have $w(k) \sim \operatorname{Lap}(0, b / \epsilon)^{r}$ so that

$$
\operatorname{var}[w(k)]=\frac{W}{\epsilon^{2}}
$$

where $W:=W\left(\Delta_{1} g, \Delta_{1} g_{x_{i}}, B\right)$ is a constant that depends upon the systems of interest, $g$ and $g_{x_{i}}$, and the adjacency parameter, $B$. 
Returning to Equation (23) and substituting in Equation (24) we find

$$
\mathbb{E}\left[\left\|z(k+1)-\xi_{k}\right\|^{2}\right] \leq \theta_{k}\left(1+\alpha_{k} \gamma_{k}\right)\left\|z(k)-\xi_{k-1}\right\|^{2}+\theta_{k} \rho_{k}+\gamma_{k}^{2} \frac{W}{\epsilon^{2}}
$$

The additive term $\gamma_{k}^{2} \frac{W}{\epsilon^{2}}$ is the only term in which the privacy parameter $\epsilon$ appears, and this term can be regarded as a penalty on convergence because it allows the expected error $\mathbb{E}[\| z(k+$ $\left.1)-\xi_{k} \|^{2}\right]$ to grow from $\left\|z(k)-\xi_{k-1}\right\|$. Viewing this term as a convergence penalty then reveals a fundamental trade-off between privacy and convergence: implementing $\epsilon$-differential privacy comes at the cost of a convergence penalty proportional to $1 / \epsilon^{2}$. We state this trade-off succinctly and informally by writing

$$
\operatorname{Privacy}(\epsilon) \Longleftrightarrow \operatorname{Convergence}\left(\epsilon^{-2}\right) \text {. }
$$

\section{Simulation Results}

Below we present numerical simulation results for a system with $n=10$ agents and $m=6$ constraints. We simulate both $\epsilon$ - and $(\epsilon, \delta)$-differential privacy.

\section{A. Example Problem}

Let there be $n=10$ agents, each with state $x_{i} \in \mathbb{R}^{2}$ and using ensemble objective function

$$
\begin{gathered}
f(x)=\left(\left(x_{1,1}-5\right)+\left(x_{1,2}+5\right)\right)+\left\|x_{2}\right\|^{2}+\left\|x_{3}-\left(\begin{array}{r}
-7 \\
7
\end{array}\right)\right\|^{2} \\
+\left(\left(x_{4,1}-8\right)+\left(x_{4,2}-8\right)\right)+\left\|x_{5}+\left(\begin{array}{l}
3 \\
3
\end{array}\right)\right\|^{4} \\
+\left(\left(x_{6,1}-10\right)+\left(x_{6,2}-10\right)\right)+\left(\left(x_{7,1}+10\right)+\left(x_{7,2}+10\right)\right) \\
+\left\|x_{8}+\left(\begin{array}{c}
7 \\
0
\end{array}\right)\right\|^{2}+\left(\left(x_{9,1}-6\right)+x_{9,2}\right)+\left\|x_{10}-\left(\begin{array}{l}
0 \\
8
\end{array}\right)\right\|^{4},
\end{gathered}
$$

where $x_{i, j}$ is the $j^{\text {th }}$ state of agent $i$ and the per-agent objectives can be discerned in the obvious way. The constraints on the agents are 


$$
g(x)=\left(\begin{array}{c}
\left\|x_{1}\right\|^{2}+\left\|x_{2}\right\|^{2}+\left\|x_{3}\right\|^{2}-10 \\
\left\|x_{4}\right\|^{2}+\left\|x_{5}\right\|^{2}+\left\|x_{6}\right\|^{2}-50 \\
\left\|x_{7}\right\|^{2}+\left\|x_{8}\right\|^{2}+\left\|x_{9}\right\|^{2}-50 \\
x_{1,1}^{2}+x_{5,1}+x_{10,1}^{2}-50 \\
x_{4,2}^{2}+x_{7,1}+x_{9,2}-20 \\
\left\|x_{8}\right\|^{2}+\left\|x_{6}\right\|^{2}-30
\end{array}\right) \leq 0 .
$$

Each agent was also constrained to lie in the box $X_{i}=[-10,10] \times[-10,10]$. The Lipschitz constants of $g$ were computed to be $K_{1}^{g}=39.82$ and $K_{2}^{g}=56.71$. The Lipschitz constants for each $g_{x_{i}}$ are shown in Table I.

\begin{tabular}{|c|c|c|}
\hline$i$ & $K_{1}^{i}$ & $K_{2}^{i}$ \\
\hline \hline 1 & 4 & $\sqrt{8}$ \\
\hline 2 & 2 & 2 \\
\hline 3 & 2 & 2 \\
\hline 4 & 2 & 2 \\
\hline 5 & 2 & 2 \\
\hline 6 & 4 & $\sqrt{8}$ \\
\hline 7 & 2 & 2 \\
\hline 8 & 4 & $\sqrt{8}$ \\
\hline 9 & 2 & 2 \\
\hline 10 & 2 & 2 \\
\hline
\end{tabular}

TABLE I

VALUES OF $K_{1}^{i}$ AND $K_{2}^{i}$ FOR $g_{x_{i}}, i \in\{1, \ldots, 10\}$

In both simulation runs below, the step-size rule discussed at the end of Section $[\mathrm{IV}]$ was used with the values

$$
\bar{\alpha}=0.1, \bar{\gamma}=0.01, c_{1}=0.3, \text { and } c_{2}=0.52,
$$

and all states and Kuhn-Tucker multipliers were initialized to zero, i.e., $x_{i}(0)=0$ for all $i \in I$ and $\mu(0)=0$. 


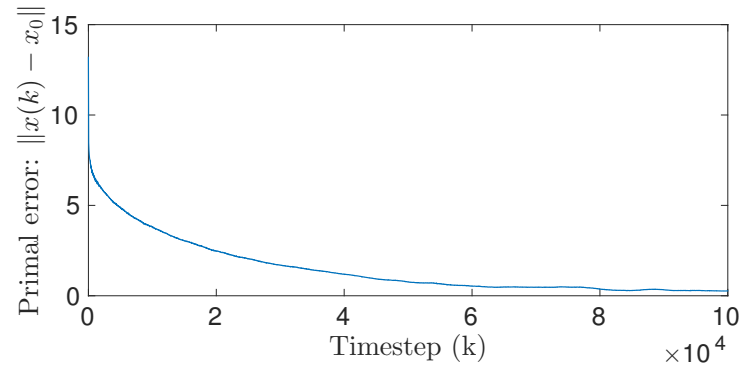

Fig. 1. The values of $\left\|x(k)-x_{0}\right\|_{2}$ for $k=1, \ldots, 100,000$ under $\epsilon$-differential privacy with Algorithm 2 The steady, monotone descent toward $x_{0}$ indicates numerical convergence to $x_{0}$ in the presence of noise.

\section{B. Simulation of $\epsilon$-differential privacy}

The adjacency parameter was chosen to be $B=1$. The value $\epsilon=\ln 2$ was used for all systems. The distribution and variance of each entry of each noisy signal were computed and are listed in Table II where we use the notation for the PDF of a random scalar with the understanding that each entry of the random matrices $w_{i}$ was generated using such a distribution.

\begin{tabular}{|c|c|c|}
\hline Noise & Distribution & Variance \\
\hline \hline$w_{1}$ & $\operatorname{Lap}(0,5.771)$ & 66.60 \\
\hline$w_{2}$ & $\operatorname{Lap}(0,2.885)$ & 16.65 \\
\hline$w_{3}$ & $\operatorname{Lap}(0,2.885)$ & 16.65 \\
\hline$w_{4}$ & $\operatorname{Lap}(0,2.885)$ & 16.65 \\
\hline$w_{5}$ & $\operatorname{Lap}(0,2.885)$ & 16.65 \\
\hline$w_{6}$ & $\operatorname{Lap}(0,5.771)$ & 66.60 \\
\hline$w_{7}$ & $\operatorname{Lap}(0,2.885)$ & 16.65 \\
\hline$w_{8}$ & $\operatorname{Lap}(0,5.771)$ & 66.60 \\
\hline$w_{9}$ & $\operatorname{Lap}(0,2.885)$ & 16.65 \\
\hline$w_{10}$ & $\operatorname{Lap}(0,2.885)$ & 16.65 \\
\hline
\end{tabular}

TABLE II

NOISY SIGNALS AND THEIR DISTRIBUTIONS FOR $\epsilon$-DIFFERENTIAL PRIVACY

The distribution for $w_{g}$ was $\operatorname{Lap}(0,57.45)$ with variance $6.600 \cdot 10^{3}$. Using this problem formulation, Algorithm 2 was run for 100,000 iterations. To show the behavior of the algorithm over time, the least-norm saddle point of $L, z_{0}=\left(x_{0}, \mu_{0}\right)$, was computed ahead of time and the values of $\left\|x(k)-x_{0}\right\|_{2}$ and $\left\|\mu(k)-\mu_{0}\right\|_{2}$ are shown in Figures 1 and 2 respectively, for 


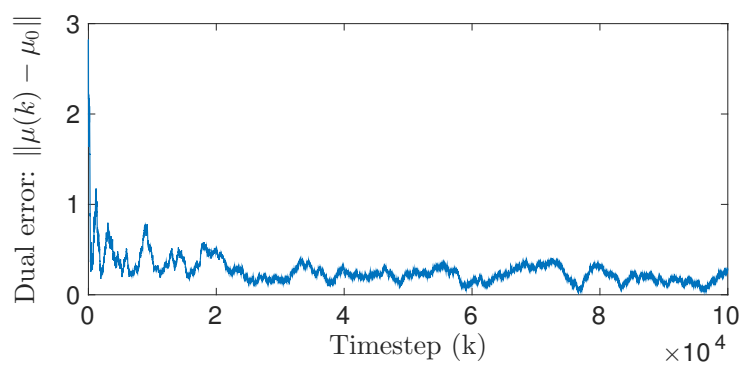

Fig. 2. The values of $\left\|\mu(k)-\mu_{0}\right\|_{2}$ for $k=1, \ldots, 100,000$ under $\epsilon$-differential privacy with Algorithm 2 Here we see an initial descent followed by a period of oscillations as $\mu(k)$ approaches $\mu_{0}$.

$1 \leq k \leq 100,000^{2}$

In Figures 1 and 2 we see a clear decreasing trend in both $\left\|x(k)-x_{0}\right\|_{2}$ and $\left\|\mu(k)-\mu_{0}\right\|_{2}$, with the primal error appearing to be monotonically decreasing and the dual error oscillating while showing a general decreasing trend. The oscillations seen are expected given that the variance of the noises added is constant while $G$ decreases in magnitude as the saddle point $z_{0}$ is approached. In fact, it is known that descent will be achieved in a gradient method as long as the norm of noise added to the gradient is less than the norm of the gradient itself [44]. In light of this fact, the trends seen in Figures 1 and 2 are not surprising because the gradients in $G$ in Algorithm 2 will have large norms far from $z_{0}$, thereby allowing them to "overpower" the noise added, while close to $z_{0}$ their norms will be smaller and the noise can dominate, causing increases in the distance to $z_{0}$ at some timesteps. Of course, $z(k) \rightarrow z_{0}$ asymptotically because these increases in $\left\|z(k)-z_{0}\right\|_{2}$ average out over very long periods of time.

The initial error values here were

$$
\left\|x(0)-x_{0}\right\|_{2}=13.19 \text { and }\left\|\mu(0)-\mu_{0}\right\|_{2}=2.169
$$

\footnotetext{
${ }^{2}$ Though the 1-norm is used for other aspects of $\epsilon$-differential privacy, we measure distance to $z_{0}$ using the 2 -norm to allow for meaningful visual comparison of the plots corresponding to $\epsilon$-differential privacy in this subsection to those corresponding to $(\epsilon, \delta)$-differential privacy in the next subsection.
} 


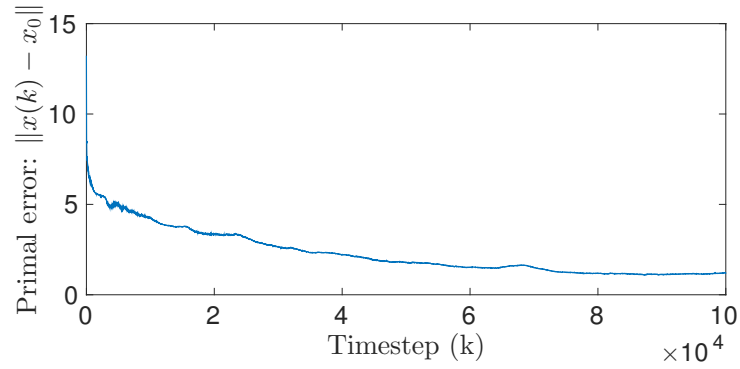

Fig. 3. The values of $\left\|x(k)-x_{0}\right\|_{2}$ for $k=1, \ldots, 100,000$ under $(\epsilon, \delta)$-differential privacy with Algorithm 2 The rapid descent toward $x_{0}$ and clear decreasing trend thereafter indicate numerical convergence to $x_{0}$ in the presence of noise.

And in this run the final error values were

$$
\left\|x(100,000)-x_{0}\right\|_{2}=0.2706 \text { and }\left\|\mu(100,000)-\mu_{0}\right\|_{2}=0.2842
$$

with these values after half of the total runtime being

$$
\left\|x(50,000)-x_{0}\right\|_{2}=0.7658 \text { and }\left\|\mu(50,000)-\mu_{0}\right\|_{2}=0.2225 \text {. }
$$

These values confirm what can be seen visually in Figures 1 and 2; shorter runtimes than 100,000 timesteps can be used while ending at a reasonable distance from $z_{0}$ and, in light of the large variances of some noises present, reasonable numbers of iterations produce an approach toward $z_{0}$ that would be useful in many applications.

\section{Simulation of $(\epsilon, \delta)$-differential privacy}

In this case the adjacency parameter was chosen to be $B>1$. The values $\epsilon=\ln 2$ and $\delta=0.01$ were used for all systems, giving $\kappa(\delta, \epsilon)=3.559$. Using this privacy policy, the distribution and variance of each noisy signal were computed and are listed in Table III.

The distribution for $w_{g}$ was $\mathcal{N}\left(0_{6 \times 1}, 4.073 \cdot 10^{4} I_{6 \times 6}\right)$ with variance $4.073 \cdot 10^{4}$. In Table III we record the distribution of each entry of the matrices $w_{i}, i \in I$, with the understanding that each $w_{i}$ has i.i.d. entries.

Using this problem formulation, Algorithm 2 was run for 100, 000 iterations and the values of $\left\|x(k)-x_{0}\right\|_{2}$ and $\left\|\mu(k)-\mu_{0}\right\|_{2}$ for $1 \leq k \leq 100,000$ are plotted in Figures 3 and 4 , respectively. 


\begin{tabular}{|c|c|c|}
\hline Noise & Distribution & Variance \\
\hline \hline$w_{1}$ & $\mathcal{N}(0,101.3)$ & 101.3 \\
\hline$w_{2}$ & $\mathcal{N}(0,50.66)$ & 50.66 \\
\hline$w_{3}$ & $\mathcal{N}(0,50.66)$ & 50.66 \\
\hline$w_{4}$ & $\mathcal{N}(0,50.66)$ & 50.66 \\
\hline$w_{5}$ & $\mathcal{N}(0,50.66)$ & 50.66 \\
\hline$w_{6}$ & $\mathcal{N}(0,101.3)$ & 101.3 \\
\hline$w_{7}$ & $\mathcal{N}(0,50.66)$ & 50.66 \\
\hline$w_{8}$ & $\mathcal{N}(0,101.3)$ & 101.3 \\
\hline$w_{9}$ & $\mathcal{N}(0,50.66)$ & 50.66 \\
\hline$w_{10}$ & $\mathcal{N}(0,50.66)$ & 50.66 \\
\hline
\end{tabular}

TABLE III

NOISY SIGNALS AND THEIR DISTRIBUTIONS FOR $(\epsilon, \delta)$-DIFFERENTIAL PRIVACY

In Figures 3 and 4 we see a similar trend to Figures 1 and 2 ; steady, monotone decrease in the primal error, and general decreases in the dual error with noticeable oscillations present.

The initial error values for this run were

$$
\left\|x(0)-x_{0}\right\|_{2}=13.19 \text { and }\left\|\mu(0)-\mu_{0}\right\|_{2}=2.169
$$

The final error values here were

$$
\left\|x(100,000)-x_{0}\right\|_{2}=1.1965 \text { and }\left\|\mu(100,000)-\mu_{0}\right\|_{2}=0.7413
$$

while after half of the total timesteps taken these values were

$$
\left\|x(50,000)-x_{0}\right\|_{2}=1.7857 \text { and }\left\|\mu(50,000)-\mu_{0}\right\|_{2}=0.2500
$$

indicating a rapid initial descent towards $z_{0}$ and close proximity to it thereafter.

Both simulation examples show a rapid decrease in the distance from $z(k)$ to $z_{0}$. Such a rapid decrease lends itself to use of this algorithm in practical applications because it allows for useful improvements to be made in the value of $f$ in a reasonable runtime while respecting the set and functional constraints of the problem. The theoretical and simulation results presented here 


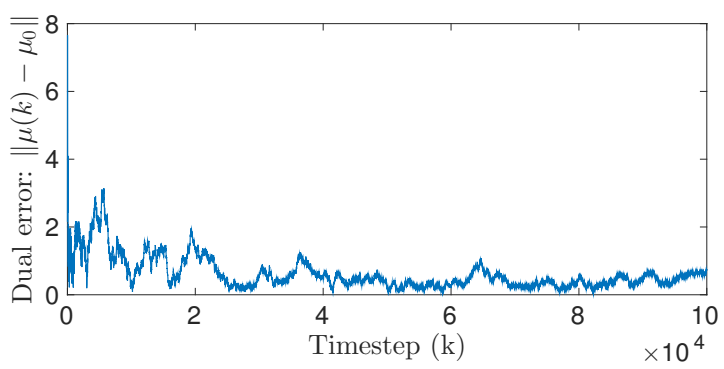

Fig. 4. The values of $\left\|\mu(k)-\mu_{0}\right\|_{2}$ for $k=1, \ldots, 100,000$ under $(\epsilon, \delta)$-differential privacy with Algorithm 2 The initial approach toward $\mu_{0}$ and oscillations in distance beyond that point indicate numerical convergence to $\mu_{0}$ when noise is added for differential privacy.

demonstrate the utility of the iterative Tikhonov regularization, even in the presence of noise with large variance. This robustness is further supported by the simulation results in [28] and demonstrates that in a practical setting strong, quantifiable guarantees of privacy can be achieved while providing useful convergence guarantees in the optimization problem of interest. Critical to the success of these numerical results is all noise being zero mean, and it is a feature of differential privacy that zero mean noise is effective at protecting sensitive information.

\section{CONCLUSION}

A differentially private optimization algorithm for teams of many agents coordinated by a central cloud computer was presented. This problem was treated as a stochastic variational inequality and solved using a Tikhonov-regularized Goldstein-Levitin-Polyak iteration. Its convergence was shown for both $\epsilon$ - and $(\epsilon, \delta)$-differential privacy and numerical convergence of the algorithm was shown in simulation, demonstrating the ability to arrive at a collective decision while maintaining privacy for the users involved in making it.

\section{REFERENCES}

[1] F. Kelly, A. Maulloo, and D. Tan, "Rate control in communication networks: shadow prices, proportional fairness and stability," in Journal of the Operational Research Society, vol. 49, 1998. [Online]. Available: citeseer.ist.psu.edu/kelly98rate.html

[2] M. Chiang, S. Low, A. Calderbank, and J. Doyle, "Layering as optimization decomposition: A mathematical theory of network architectures," Proceedings of the IEEE, vol. 95, no. 1, pp. 255-312, Jan 2007. 
[3] J. Cortes, S. Martinez, T. Karatas, and F. Bullo, "Coverage control for mobile sensing networks," in Robotics and Automation, 2002. Proceedings. ICRA'02. IEEE International Conference on, vol. 2. IEEE, 2002, pp. 1327-1332.

[4] D. E. Soltero, M. Schwager, and D. Rus, "Decentralized path planning for coverage tasks using gradient descent adaptive control," The International Journal of Robotics Research, 2013.

[5] S. Boyd, N. Parikh, E. Chu, B. Peleato, and J. Eckstein, "Distributed optimization and statistical learning via the alternating direction method of multipliers," Found. Trends Mach. Learn., vol. 3, no. 1, pp. 1-122, Jan. 2011.

[6] M. Khan, G. Pandurangan, and V. Kumar, "Distributed algorithms for constructing approximate minimum spanning trees in wireless sensor networks," Parallel and Distributed Systems, IEEE Transactions on, vol. 20, no. 1, pp. 124-139, Jan 2009.

[7] N. Trigoni and B. Krishnamachari, "Sensor network algorithms and applications Introduction," Philosophical Transactions of the Royal Scoeity A - Mathematical, Physical, and Engineering Sciences, vol. 370, no. 1958, SI, pp. 5-10, JAN 13 2012.

[8] P. Vytelingum, T. D. Voice, S. D. Ramchurn, A. Rogers, and N. R. Jennings, "Agent-based micro-storage management for the smart grid," in Proceedings of the 9th International Conference on Autonomous Agents and Multiagent Systems: volume 1-Volume 1. International Foundation for Autonomous Agents and Multiagent Systems, 2010, pp. 39-46.

[9] S. Caron and G. Kesidis, "Incentive-based energy consumption scheduling algorithms for the smart grid," in Smart Grid Communications (SmartGridComm), 2010 First IEEE International Conference on, Oct 2010, pp. 391-396.

[10] M. H. Nazari, Z. Costello, M. J. Feizollahi, S. Grijalva, and M. Egerstedt, "Distributed frequency control of prosumer-based electric energy systems," Power Systems, IEEE Transactions on, vol. 29, no. 6, pp. 2934-2942, 2014.

[11] A. Nedic and A. Ozdaglar, "Distributed subgradient methods for multi-agent optimization," Automatic Control, IEEE Transactions on, vol. 54, no. 1, pp. 48-61, Jan 2009.

[12] I. Lobel and A. Ozdaglar, "Distributed subgradient methods for convex optimization over random networks," Automatic Control, IEEE Transactions on, vol. 56, no. 6, pp. 1291-1306, June 2011.

[13] E. Wei, A. Ozdaglar, and A. Jadbabaie, "A distributed newton method for network utility maximization I: Algorithm," Automatic Control, IEEE Transactions on, vol. 58, no. 9, pp. 2162-2175, 2013.

[14] D. P. Bertsekas and J. N. Tsitsiklis, Parallel and Distributed Computation: Numerical Methods. Upper Saddle River, NJ, USA: Prentice-Hall, Inc., 1989.

[15] United States Department of Energy Office of the General Counsel, "Data access and privacy issues related to smart grid technologies," United States, Tech. Rep., October 2010.

[16] The European Data Protection Supervisor, "Opinion of the european data protection supervisor on the commission recommendation on preparations for the roll-out of smart metering systems,” European Union, Tech. Rep., June 2012.

[17] C. Dwork, "Differential privacy," in 33rd International Colloquium on Automata, Languages and Programming, Part II (ICALP 2006), ser. Lecture Notes in Computer Science, vol. 4052. Venice, Italy: Springer Verlag, July 2006, pp. 1-12.

[18] C. Dwork, F. McSherry, K. Nissim, and A. Smith, "Calibrating noise to sensitivity in private data analysis," in Proceedings of the Third Conference on Theory of Cryptography, ser. TCC'06. Berlin, Heidelberg: Springer-Verlag, 2006, pp. 265-284.

[19] C. Dwork, "Differential privacy: A survey of results," in Theory and Applications of Models of Computation, ser. Lecture 
Notes in Computer Science, M. Agrawal, D. Du, Z. Duan, and A. Li, Eds. Springer Berlin Heidelberg, 2008, vol. 4978, pp. 1-19.

[20] C. Dwork and A. Roth, "The algorithmic foundations of differential privacy," Theoretical Computer Science, vol. 9, no. 3-4, pp. 211-407, 2013.

[21] J. Le Ny and G. Pappas, "Differentially private filtering," Automatic Control, IEEE Transactions on, vol. 59, no. 2, pp. 341-354, Feb 2014.

[22] S. P. Kasiviswanathan and A. Smith, "A note on differential privacy: Defining resistance to arbitrary side information," CoRR abs/0803.3946, 2008.

[23] J. Hsu, A. Roth, T. Roughgarden, and J. Ullman, "Privately solving linear programs," in Automata, Languages, and Programming, ser. Lecture Notes in Computer Science, J. Esparza, P. Fraigniaud, T. Husfeldt, and E. Koutsoupias, Eds. Springer Berlin Heidelberg, 2014, vol. 8572, pp. 612-624.

[24] S. Han, U. Topcu, and G. Pappas, "Differentially private convex optimization with piecewise affine objectives," in Decision and Control (CDC), 2014 IEEE 53rd Annual Conference on, Dec 2014, pp. 2160-2166.

[25] Z. Huang, S. Mitra, and N. Vaidya, "Differentially private distributed optimization," in Proceedings of the 2015 International Conference on Distributed Computing and Networking, ser. ICDCN '15. New York, NY, USA: ACM, 2015, pp. 4:1-4:10.

[26] Z. Huang, S. Mitra, and G. Dullerud, "Differentially private iterative synchronous consensus," in Proceedings of the 2012 ACM Workshop on Privacy in the Electronic Society, ser. WPES '12. New York, NY, USA: ACM, 2012, pp. 81-90.

[27] Q. Zhang, L. Cheng, and R. Boutaba, "Cloud computing: state-of-the-art and research challenges," Journal of Internet Services and Applications, vol. 1, no. 1, pp. 7-18, 2010.

[28] M. Hale and M. Egerstedt, "Differentially private cloud-based multi-agent optimization with constraints," in American Control Conference (ACC), 2015, July 2015, pp. 1235-1240.

[29] D. P. Bertsekas, A. E. Ozdaglar, and A. Nedi, Convex analysis and optimization, ser. Athena scientific optimization and computation series. Belmont, Massachusetts: Athena Scientific, 2003.

[30] H. Uzawa, "Iterative methods for concave programming," Studies in linear and nonlinear programming, vol. 6, 1958.

[31] R. T. Rockafellar, "On the maximal monotonicity of subdifferential mappings." Pacific J. Math., vol. 33, no. 1, pp. 209-216, 1970. [Online]. Available: http://projecteuclid.org/euclid.pjm/1102977253

[32] I. Konnov, Equilibrium models and variational inequalities. Elsevier, 2007, vol. 210.

[33] F. Facchinei and J.-S. Pang, Finite-dimensional variational inequalities and complementarity problems. Springer Verlag, 2003, vol. 1 and 2 .

[34] A. Bakushinskii and B. Polyak, "Solution of variational inequalities," Doklady Akademii Nauk SSSR, vol. 219, no. 5, pp. 1038-1041, 1974.

[35] B. Poljak, "Nonlinear programming methods in the presence of noise," Mathematical programming, vol. 14, no. 1, pp. 87-97, 1978.

[36] A. A. Goldstein, "Convex programming in hilbert space," Bull. Amer. Math. Soc., vol. 70, no. 5, pp. 709-710, 091964. [Online]. Available: http://projecteuclid.org/euclid.bams/1183526263

[37] E. Levitin and B. Polyak, "Constrained minimization methods," \{USSR $\}$ Computational Mathematics and Mathematical 
Physics, vol. 6, no. 5, pp. 1 - 50, 1966. [Online]. Available: http://www.sciencedirect.com/science/article/pii/ 0041555366901145

[38] C. Dwork, K. Kenthapadi, F. McSherry, I. Mironov, and M. Naor, "Our data, ourselves: Privacy via distributed noise generation," in Advances in Cryptology (EUROCRYPT 2006), ser. Lecture Notes in Computer Science, vol. $4004 . \quad$ Saint Petersburg, Russia: Springer Verlag, May 2006, p. 486503.

[39] F. Yousefian, A. Nedic, and U. Shanbhag, "A regularized smoothing stochastic approximation (RSSA) algorithm for stochastic variational inequality problems," in Simulation Conference (WSC), 2013 Winter, Dec 2013, pp. 933-944.

[40] J. Koshal, A. Nedic, and U. Shanbhag, "Regularized iterative stochastic approximation methods for stochastic variational inequality problems," Automatic Control, IEEE Transactions on, vol. 58, no. 3, pp. 594-609, March 2013.

[41] B. T. Poljak, Introduction to optimization. Optimization Software, 1987.

[42] H. M. Edwards, Riemann's zeta function. Courier Corporation, 2001, vol. 58.

[43] D. H. Greene and D. E. Knuth, Mathematics for the analysis of algorithms, ser. Progress in computer science. Boston, Basel, Stuttgart: Birkhauser, 1981. [Online]. Available: http://opac.inria.fr/record=b1116524

[44] G. Zoutendijk, "Nonlinear programming, computational methods," in Integer and Nonlinear Programming, J. Abadie, Ed. North-Holland, 1970, pp. 37-86. 